\title{
Changes in cellular immune responses of Chilo suppressalis Walker (Lepidoptera: Crambidae) due to pyriproxyfen treatment
}

\author{
Seyyedeh Kimia Mirhaghparast ${ }^{1}$, Arash Zibaee ${ }^{1 *}$, Hassan Hoda ${ }^{2}$, Jalal Jalali Sendi ${ }^{1}$ \\ ${ }^{1}$ Department of Plant Protection, Faculty of Agricultural Sciences, University of Guilan, Rasht, 416351314 Iran \\ ${ }^{2}$ Biological Control Department, Iranian Institute of Plant Protection, Amol, 91951-46191 Iran
}

Received: March 23, 2015

Accepted: June 17, 2015

\begin{abstract}
The effects of pyriproxyfen were determined on the cellular immunity and phenoloxidase activity in the 4th instar larvae of Chilo suppressalis Walker. The bioassay results revealed the effective concentrations of: 10L:18C, 30L:72C and 50L:190C $\mu \mathrm{g} \cdot \mathrm{ml}^{-1}$. The sole effect of 18 and $72 \mu \mathrm{g} \cdot \mathrm{ml}^{-1}$ concentrations at intervals of $1-3 \mathrm{~h}$ caused a higher number of total hemocytes in the treated larvae than the control, but the reverse results were observed after 6-24 h. The number of plasmatocytes was lower than that of the control for intervals of 3-24 $\mathrm{h}$ but the number of granulocytes was higher than the control after 1-3 $\mathrm{h}$ although no significant differences were observed at the other times. In the treated larvae, the activities of phenoloxidase were higher and lower than those of the control after 1-3 h and 6-24 h, respectively. The combined effects of pyriproxyfen and the entomopathogenic fungus, Beauveria bassiana isolate B3 caused higher numbers of total hemocytes, plasmatocytes, and granulocytes in the treated larvae by use of the three concentrations of pyriproxyfen, at intervals of 6 and $12 \mathrm{~h}$. Although the numbers of nodules in the larvae treated with concentrations of $18 \mu \mathrm{g} \cdot \mathrm{ml}^{-1} \mathrm{were}$ higher than those of other treatments, the overall numbers were lower than those of the control. Finally, the activity of phenoloxidase in the treated larvae was higher than that of the control, at intervals of 6 and $12 \mathrm{~h}$ post-treatment. Findings of the current study indicate an intervening role of pyriproxyfen in the cellular immunity of $C$. suppressalis to entomopathogenic objects.
\end{abstract}

Key words: Beauveria bassiana, Chilo suppressalis, hemocyte, immune response, pyriproxyfen

\section{Introduction}

The juvenile hormones (JHs) are the products of corpora allata, in insects that belong to the family of acyclic sesquiterpenoids. Researchers have identified JHs in approximately 100 insect species from at least 10 insect orders (Goodman and Granger 2005). These compounds may be involved in the control of gonadal development, vitellogenin synthesis, regulation of metamorphosis, caste determination, behavior, diapause, and various polyphenisms (Nijhout 1994). Hence, the alteration in titer of JHs causes severe physiological and behavioral disruptions leading to death or malformation. The importance of JHs in the physiological processes of insects opened a new area to synthesis several compounds called JH analogs. Pyriproxyfen, 4-phenoxyphenyl (RS)-2-(2-pyridyloxy) propyl ether, is a pyridine-based insecticide which acts as an insect growth regulator against cockroaches, fleas, lepidopteran, coleopteran, and other pests. Ishaaya and Horowitz (1992) suggested that pyriproxyfen mimics the action of the juvenile hormones on a number of physiological processes but it is a potent inhibitor of embryogenesis, metamorphosis, and adult formation.

Chilo suppressalis Walker (Lepidoptera: Crambidae) is a serious pest of rice in different regions. The larvae of this pest intensively feed on the stems. The three overlapping generations of the pest cause severe yield loss an- nually. Here in Iran, light traps and intensive spraying of pesticides such as diazinon, are the main control procedures to decrease population outbreaks of $C$. suppressalis. Resistance of $C$. suppressalis to diazinon in several areas of northern Iran was reported by Zibaee et al. (2009). Resistance to the pesticides used, and environmental concerns of chemical spraying, require changing the control procedures to safer ones. Recently, Ramzi and Zibaee (2014) determined the virulence of various entomopathogenic fungi against $C$. suppressalis larvae. The above-noted authors performed bioassays, biochemical analysis, and cluster analysis which indicated that isolate BB3 was the most effective fungus against the larvae. In another study, Zibaee and Malagoli (2014) studied the cellular immune responses of $C$. suppressalis in the presence of several entomopathogenic fungi; three isolates of Beauveria bassiana, Metarhizium anisopliae, Isaria fumosoroseus, and Lecanicilium lecanii. Prohemocytes, granulocytes, plasmatocytes, and oenocytoids were identified as the main circulating hemocytes in the hemolymph of larvae by the major roles of granulocytes and plasmatocytes. Also, the number of hemocytes, and the phenoloxidase activity, showed fluctuating results after injection of the entomopathogenic fungi. Although C. suppressalis, like other insects, could overcome entomopathogenic organisms via cellular and humeral responses, there are several factors which could 
modulate immunity. These factors include hormones, feeding status, developmental stages, and temperature. The determination of modulating factors on insect immunity could elucidate the ability or deficiency of an insect vs. non-self-objects (Zibaee et al. 2009). Juvenile hormone and its analogs are factors affecting the immunity of insects, mainly by altering the number of immunocytes (Gelbič et al. 2005; Franssens et al. 2006; Zibaee et al. 2012; Rahimi et al. 2013). Apart from immune responses, Mirhaghparast et al. (2014) found disturbance effects of pyriproxyfen on several enzymatic and non-enzymatic components engaged in intermediary metabolism of C. suppressalis. Regarding the impressive effects of pyriproxyfen on these processes, the positive or negative effects of the JH analogs (e.g. pyriproxyfen) on the cellular immunity of $C$. suppressalis larvae showing possible compatibility with biological control using B. bassiana must be made clear. Hence, the current study was conducted to find out how pyriproxyfen affects cellular immunity alone or in the presence of B. bassiana spores.

\section{Materials and Methods}

\section{Chilo suppressalis rearing}

Individuals of $C$. suppressalis were reared on the Hashemi variety of rice in the Department of Biological Control, the Iranian Institute of Plant Protection, Amol. Rice varieties were planted in buckets (height $40 \mathrm{~cm}$, width $20 \mathrm{~cm}$ ) under cages in greenhouse. After rearing for three generations, egg patches were transferred to a laboratory, where the conditions were: $25^{\circ} \mathrm{C}, 85 \%$ of relative humidity $(\mathrm{RH})$ and $16 \mathrm{~L}$ : $8 \mathrm{D}$ of photoperiod. These egg patches were then reared to 4 th larval instars.

\section{Beauveria bassiana culture}

Based on Ramzi and Zibaee (2014), the BB3 isolate of $B$. bassiana showing the highest virulence on C. suppressalis was cultured at $25^{\circ} \mathrm{C}\left( \pm 1^{\circ} \mathrm{C}\right)$ on Potato Dextrose Agar (pH 5.6) amended with $1 \%$ yeast extract. After 14 days, the conidia were washed off with a $0.01 \%$ solution of Tween 80 (Sigma Aldrich, USA), and a concentration of $10^{4}$ spore $\cdot \mathrm{ml}^{-1}$ was prepared for a combined exposure using pyriproxyfen (Zibaee and Malagoli 2014).

\section{Bioassay of pyriproxyfen}

Different concentrations of pyriproxyfen including 50, 100, 300,500 , and $1,000 \mu \mathrm{g} \cdot \mathrm{ml}^{-1}$, were prepared and $2 \mu \mathrm{l}$ of each of the concentrations was topically exposed to 4 th larval instars. The mortality of the larvae was recorded during the $72 \mathrm{~h}$ period. Then, the data were inserted in POLOPC software to calculate the $\mathrm{LC}_{10}, \mathrm{LC}_{30}$, and $\mathrm{LC}_{50}$ values. The bioassay was done using three replicates containing 10 larvae in each, together with the control $(\mathrm{N}=180)$.

\section{Effect of pyriproxyfen on cellular immunity}

Larvae of C. suppressalis (group I) were separately treated with $2 \mu \mathrm{l}$ of pyriproxyfen concentrations $(18,72$ and
$190 \mu \mathrm{g} \cdot \mathrm{ml}^{-1}$ ) to find the sole effects of pyriproxyfen on hemocyte numbers and phenoloxidase activity. The control larvae treatment used acetone (number of individuals per treatment including the control was 30 larvae; $\mathrm{N}=120$ ). Group II larvae underwent a treatment that used $2 \mu \mathrm{l}$ of pyriproxyfen concentration and then after $4 \mathrm{~h}$, these Group II larvae were injected with $10^{4}$ spore $\cdot \mathrm{ml}^{-1}$ of B. bassiana var. BB3 to find the number of hemocytes, the nodule formation, and the phenoloxidase activity. The control larvae were treated by acetone and injected with distilled water (number of individuals per treatment including the control was 30 larvae, $N=120$ ). After both experiments, the hemolymph of the larvae were collected from the first abdominal proleg at 1, 3, 6, 12, and $24 \mathrm{~h}$ intervals. The hemolymph was immediately diluted with an anticoagulant solution $(0.01 \mathrm{M}$ ethylenediamine tetraacetic acid, $0.1 \mathrm{M}$ glucose, $0.062 \mathrm{M} \mathrm{NaCl}$, and $0.026 \mathrm{M}$ citric acid, $\mathrm{pH}$ 4.6) described by Azambuja et al. (1991). The preparation proportion was $1: 4$ of anticoagulant solution and hemolymph. Samples taken from different time intervals were loaded into a hemocytometer to count the number of total hemocytes, plasmatocytes, granulocytes, and nodules. Please note the number of nodules just evaluated after the combined effects of pyriproxyfen and B. bassiana.

\section{Assay of phenoloxidase activity}

Phenoloxidase activities (PO) in the hemolymph of the control and treated larvae were assessed at different time intervals. They were assessed according to Willson et al. (2002). Briefly, $8 \mu \mathrm{l}$ of hemolymph was added to $50 \mu \mathrm{l}$ of ice-cold PBS ( $\mathrm{pH} 7$ ) and gently mixed. The samples were frozen at $-20^{\circ} \mathrm{C}$ to disrupt hemocyte membranes. The enzymatic activities were assayed spectrophotometrically using $10 \mathrm{mM}$ L-dopa (3,4-dihydroxyphenylalanine) as the substrate. Incubation was done for $5 \mathrm{~min}$ and the absorbance was then read at $492 \mathrm{~nm}$ during the linear phase of the reaction.

\section{Protein assay}

Protein concentrations were assayed according to the method described by Lowry et al. (1951). The method recruits the reaction of $\mathrm{Cu}^{2+}$, produced by the oxidation of peptide bonds with Folin-Ciocalteu reagent. In the assay, $20 \mu \mathrm{l}$ of the homogenised sample was added to $100 \mu \mathrm{l}$ of the reagent, and incubation took place for $30 \mathrm{~min}$ prior to reading the absorbance at $545 \mathrm{~nm}$ (recommended by Ziest Chem. Co., Tehran-Iran).

\section{Statistical analysis}

All data from a complete randomised design were compared by one-way analysis of variance (ANOVA) followed by Tukey's test. Differences between samples were statistically considered at a probability less than 5\% and marked in the figures and tables. The treatment values were compared in the same time intervals. 


\section{Results and Discussion}

Both pesticides and entomopathogenic organisms are considered to be efficient means for decreasing an insect population. Since Integrated Pest Management (IPM) is recommended as the rational and safe procedure to decrease the damage caused by pests, potential synergism or antagonism of the various control procedures must be determined. The effects of pesticides, mainly insect growth regulators (IGRs), must be determined to have a better background of IPM and to make way for the adaptability or inadaptability of the IGRs with other control tactics. In the current study, pyriproxyfen affected the numbers of hemocytes, the nodule formation, and the phenoloxidase activity in the C. suppressalis 4 th instar larvae, in both sole exposure and combined with the entomopathogenic fungus, B. bassiana (isolate BB3). When pyriproxyfen, in concentrations of 18 and $72 \mu \mathrm{g}$. $\cdot \mathrm{ml}^{-1}$, was solely exposed to larvae, the number of the total hemocytes (after 1-3 h) was higher than that of the control (after 1-3 h). By increasing the incubation time, the number of total hemocytes in all the treated concentrations were lower than in the control (Fig. 1A). The combined treatment of pyriproxyfen and B. bassiana led to higher numbers of total hemocytes at intervals of 6-24 h post-treatment than in the control (except for the concentration of $190 \mu \mathrm{g} \cdot \mathrm{ml}^{-1}$ after $24 \mathrm{~h}$ ). But the number of cells in the treated larvae at time intervals of 1 and $3 \mathrm{~h}$ were lower than in the control, except for the concentration of $18 \mu \mathrm{g}$. - $\mathrm{ml}^{-1}$ (Fig. 1B). Sole treatment of pyriproxyfen decreased the number of plasmatocytes in all of the time intervals in comparison with the control, except for 1 and $3 \mathrm{~h}$ post-treatment. The number of plasmatocytes in the concentration of $18 \mu \mathrm{g} \cdot \mathrm{ml}^{-1}$ tended to be higher or the same as the control (Fig. 2A). Plasmatocytes in combined effects with pyriproxyfen and B. bassiana at intervals of 6-24 h post-treatment, showed higher numbers than the control. However, the results were reversed in the two first, timeintervals (Fig. 2B). Although the numbers of granulocytes after the sole treatment of pyriproxyfen were higher than those of the control, the combined effects of pyriproxyfen and B. bassiana increased the number of these cells in comparison with the control, after 6 and $12 \mathrm{~h}$ of post-treatment (Fig. 3A, B). In other time intervals, the number of granulocytes were lower than for the control or no significant differences were observed (Fig. 3B). George and Ambrose (2004) found that treating Rhynocoris kumarii Ambrose and Livingstone (Hemiptera: Reduviidae) with organophosphate insecticides increased the total hemocyte counts, mainly granulocytes, while organochlorines decreased the total number of hemocytes, mainly granulocytes, but increased the numbers of prohemocytes and plasmatocytes. Botanical insecticides, like Azadirachtin and Artemisia annua L. (Asteraceae), decreased the number of plasmatocytes and granulocytes in Eurygaster integriceps Puton (Hemiptera: Scutelleridae) and Rhodnius
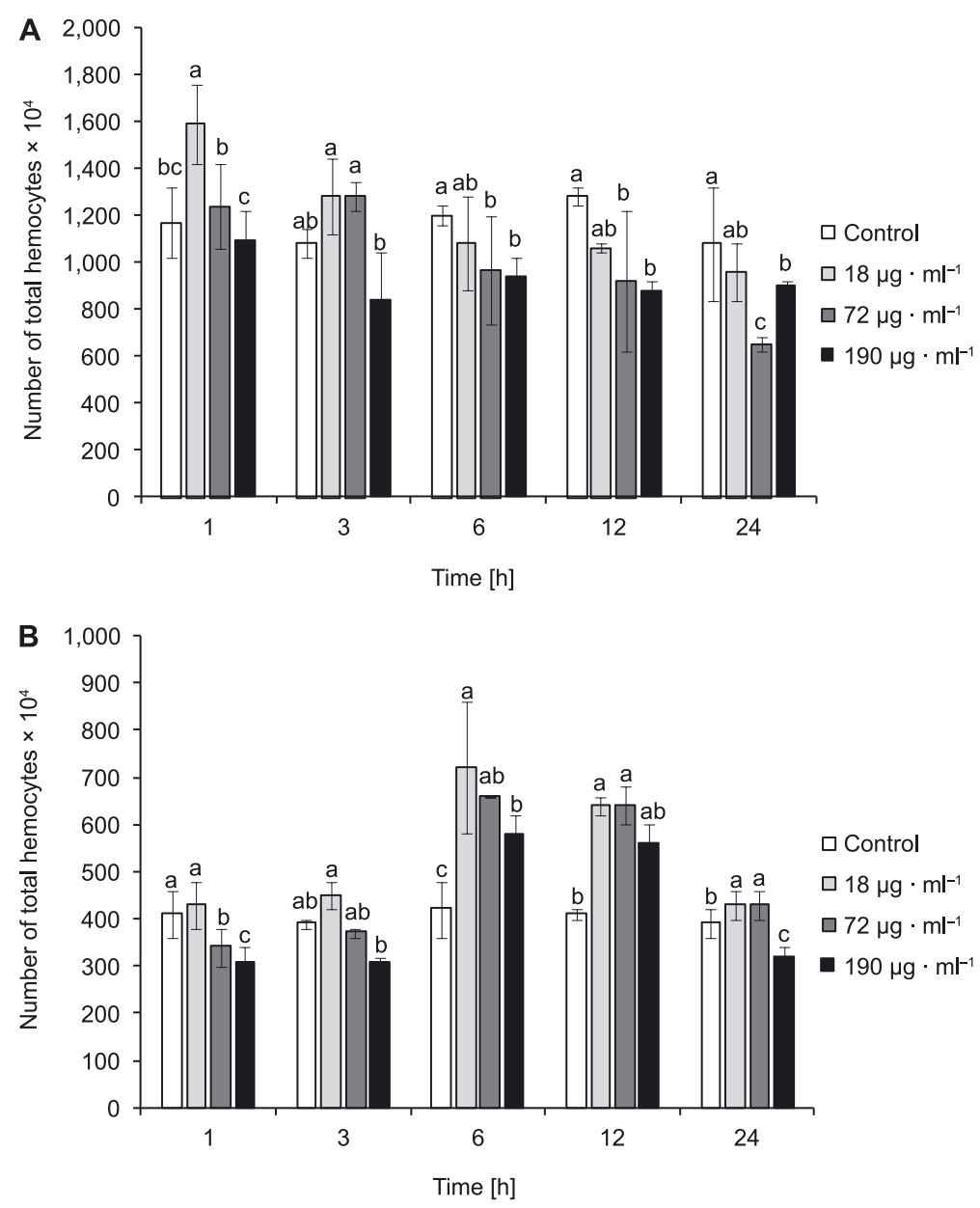

Fig. 1. Effects of pyriproxyfen (A) and pyriproxyfen + Beauveria bassiana (B) on total hemocyte counts of Chilo suppressalis. Statistical differences have been shown by various letters in each time intervals ( $\mathrm{p} \leq 0.05$, Tukey test) 

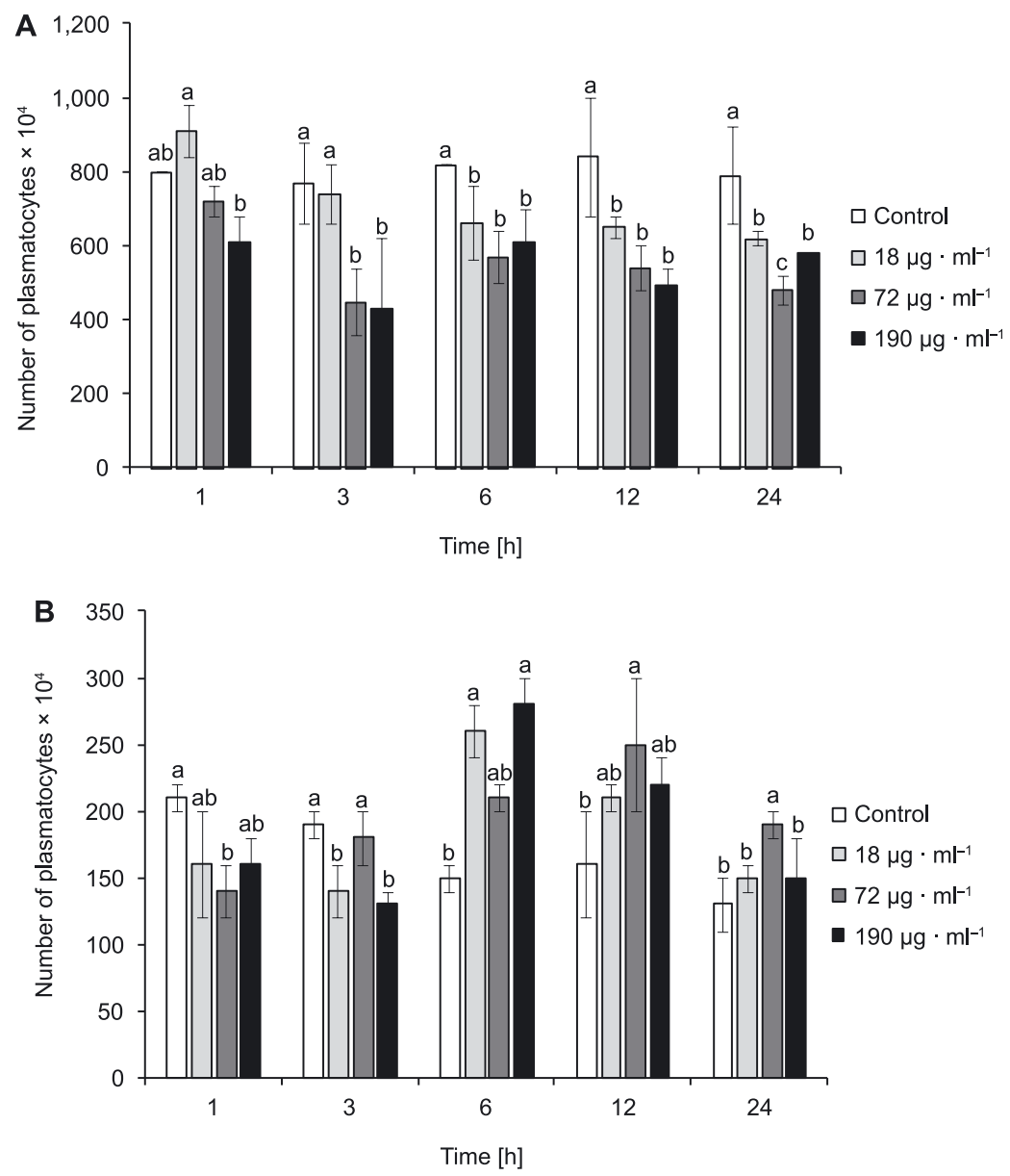

Fig. 2. Effects of pyriproxyfen (A) and pyriproxyfen + Beauveria bassiana (B) on plasmatocyte counts of Chilo suppressalis. Statistical differences have been shown by various letters in each time intervals $(p \leq 0.05$, Tukey test)

prolixus L. (Hemiptera: Reduviidae) in a dose-dependent manner (Azambuja et al. 1991; Zibaee and Bandani 2010). Insect hormones, like 20-hydroxyecdysone (20E) and JH, have shown two distinct effects on the cellular immunity of an insect. In the majority of studies, 20E caused proliferation of hemocytes, while $\mathrm{JH}$ and its analogs showed adverse results (James and Xu 2012). In their study, Gelbič et al. (2005) showed that the JH analog, metyrapone, reduced the number of granulocytes in Spodoptera littoralis Fabricius (Lepidoptera: Noctuidae), while it increased the number of plasmatocytes. Kim et al. (2008) reported an antagonistic effect of juvenile hormone on the numbers and the hemocyte-spreading behavior in Spodoptera exigua (Hübner). It was noted, in the study by Zibaee et al. (2012), that pyriproxyfen decreased the numbers of total hemocytes, plasmatocyte, and granulocyte in E. integriceps adults. Rahimi et al. (2013) demonstrated inhibitory effects of pyriproxyfen on the total hemocyte counts in addition to the numbers of plasmatocytes and granulocytes in the 4th instar larvae of Ephestia kuehniella Zeller (Lepidoptera: Pyralidae). Our overall results revealed that the sole treatment of pyriproxyfen decreased the number of total hemocytes, plasmatocytes, and granulocytes when taking into account statistical significance. There was an exception for the total hemocytes at the $1 \mathrm{~h}$ time interval at the lowest concentration. When the fungus, $B$. bassiana, was injected after the pyriproxyfen treat- ment, the highest number of hemocytes were observed after 6 and $12 \mathrm{~h}$, which was higher than that of the control. The effects of pesticides on the hemocytes of insects are different and depend on the chemical nature of the pesticides. The negative effects of JH analogs, like pyriproxyfen on the hemocyte numbers, could be attributed to cytotoxic effects, to the inhibition of larval hematopoietic function, or to cell proliferation (Zibaee et al. 2012). Inhibition studies were done by Kim et al. (2008), regarding the differences of hemocyte numbers alone, and the combined effects of pyriproxyfen. They performed the studies to have a better inderstanding of the mechanism. They reported antagonistic effects of ethoxyzolamide; a known inhibitor of the JH membrane receptor on the plasmatocytes of Spodoptera frugiperda (J.E. Smith). They suggested that $\mathrm{JH}$ and maybe their analogs have a membrane action on the plasmatocyte. Hemocytes somehow showed a response to the JH treatment. Kim et al. (2008) concluded, though, that JH utilised membrane or cytosolic receptors to exert its effects on hemocytes rather than nuclear gene expression. Moreover, some other mediators, such as eicosanoids, are activated in response to pathogen infection so they caused the hemocytes' sensitivity at points of infection, increasing cellular responses such as phagocytosis and nodule formation (Kim et al. 2008).

No nodule was observed after the sole effects of pyriproxyfen. But the combined treatment of pyriproxyfen 

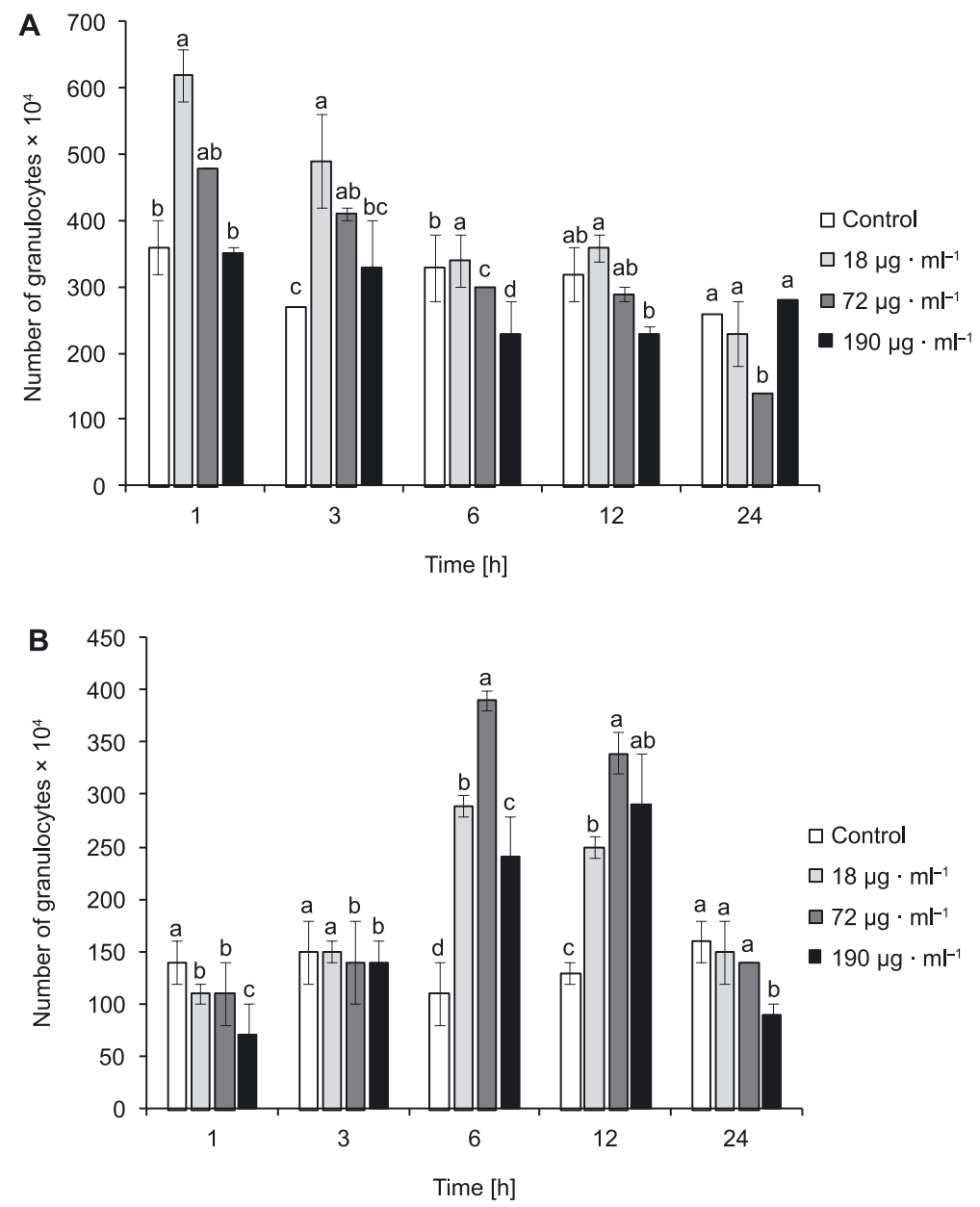

Fig. 3. Effects of pyriproxyfen (A) and pyriproxyfen + Beauveria bassiana (B) on granulocyte counts of Chilo suppressalis. Statistical differences have been shown by various letters in each time intervals ( $p \leq 0.05$, Tukey test)

and B. bassiana revealed a lower numbers of nodules in comparison with the control at all the time intervals (Fig. 4). In the two first time-intervals, the activities of phenoloxidase were higher than those of the control after pyriproxyfen treatment. However, the enzymatic activities decreased in other time intervals (Fig. 5A). The activities of the enzyme were found to be higher than those of the control, at time intervals of $6-12 \mathrm{~h}$ for the combined treatment and mainly for the concentrations of 72 and $190 \mu \mathrm{g}$. $\cdot \mathrm{ml}^{-1}$. Adverse results were observed, though, in the two, first time-intervals (Fig. 5B). Rantala et al. (2003) demonstrated suppressive effects of $\mathrm{JH}$ on both $\mathrm{PO}$ and encapsulation in Tenebrio molitor L. (Coleoptera: Tenebrionidae). It was reported by Rahimi et al. (2013) that the highest number of nodules were found at 6-12 h post injection of E. kuehniella larvae by B. bassiana, for both the control and the pyriproxyfen-treated larvae, yet the number of nodules in the treated larvae were significantly lower than those in the control. The authors found no significant differences in the case of phenoloxidase activity (Rahimi et al. 2013). It was observed by Franssens et al. (2006) that both $\mathrm{JH}$ and its analogs affected the nodulation in the larvae of $N$. bullata by reducing the ability of the larvae to form nodules in response to laminarin, along with a down regulation of phenoloxidase activity. The lower numbers of formed nodules in the pyriproxyfen-treated, C. suppressalis larvae might be because of the inhibitory effects of the insecticide on the cell proliferation, and the differentiation into macrophages. Also, since phenoloxidases are synthesised as zymogen by hemocytes, one of the reasons for the lowering of phenoloxidase activity might be the reduced numbers of hemocytes caused by pyriproxyfen. The second reason has been suggested by the works of Mirhaghparast and Zibaee (2013) and Delkash-Roudsari et al. (2015) on in vitro effects of pyriproxyfen on phenoloxidase of C. suppressalis and E. kuehniella. The results revealed significant inhibition by both extracted and purified phenoloxidase due to in vitro treatment using pyriproxyfen. So, it might be concluded that pyriproxyfen could decrease the phenoloxidase activity of insects by not only reducing the number of hemocytes but also by direct inhibition of phenoloxidase activity.

The results of the current study revealed two different effects of pyriproxyfen after a sole treatment or after a pyriproxyfen-treatment in combination with $B$. bassiana on the 4th instar larvae of $C$. suppressalis. The results revealed that a sole treatment of pyriproxyfen decreased the number of hemocytes and the nodule formation, and any positive effects would be expected in the earlier time intervals. The activity of phenoloxidase even showed a negative correlation by the effects of pyriproxyfen on the hemocytes. These finding and those of a previous study on intermediary metabolism, might indicate that there are intervening roles of this compound in the physiologi- 

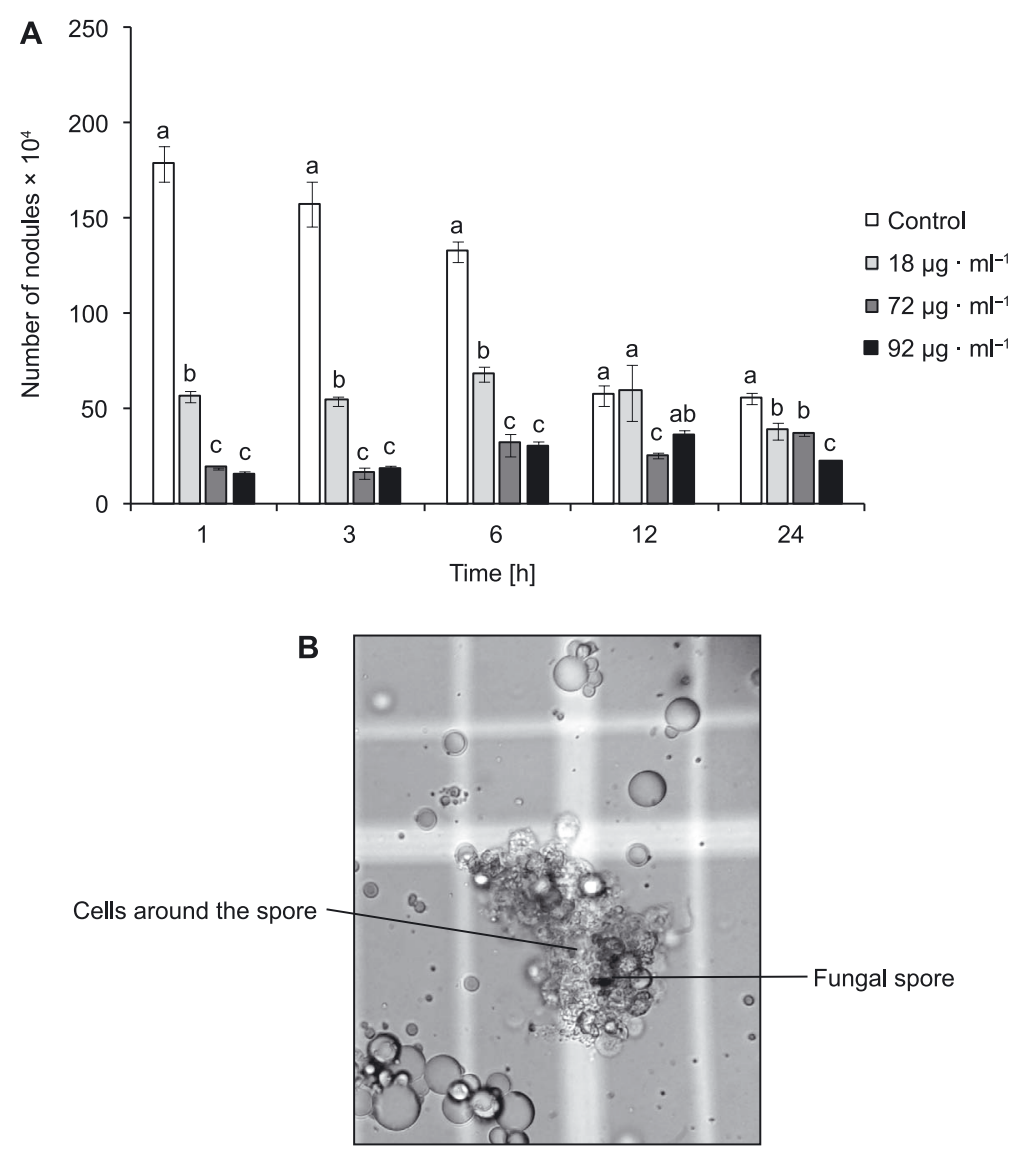

Fig. 4. Effects of pyriproxyfen + Beauveria bassiana (A) on number of nodules in Chilo suppressalis. A nodule found in the injected larvae of Ch. suppressalis (B). Statistical differences have been shown by various letters in each time intervals ( $\mathrm{p} \leq 0.05$, Tukey test)
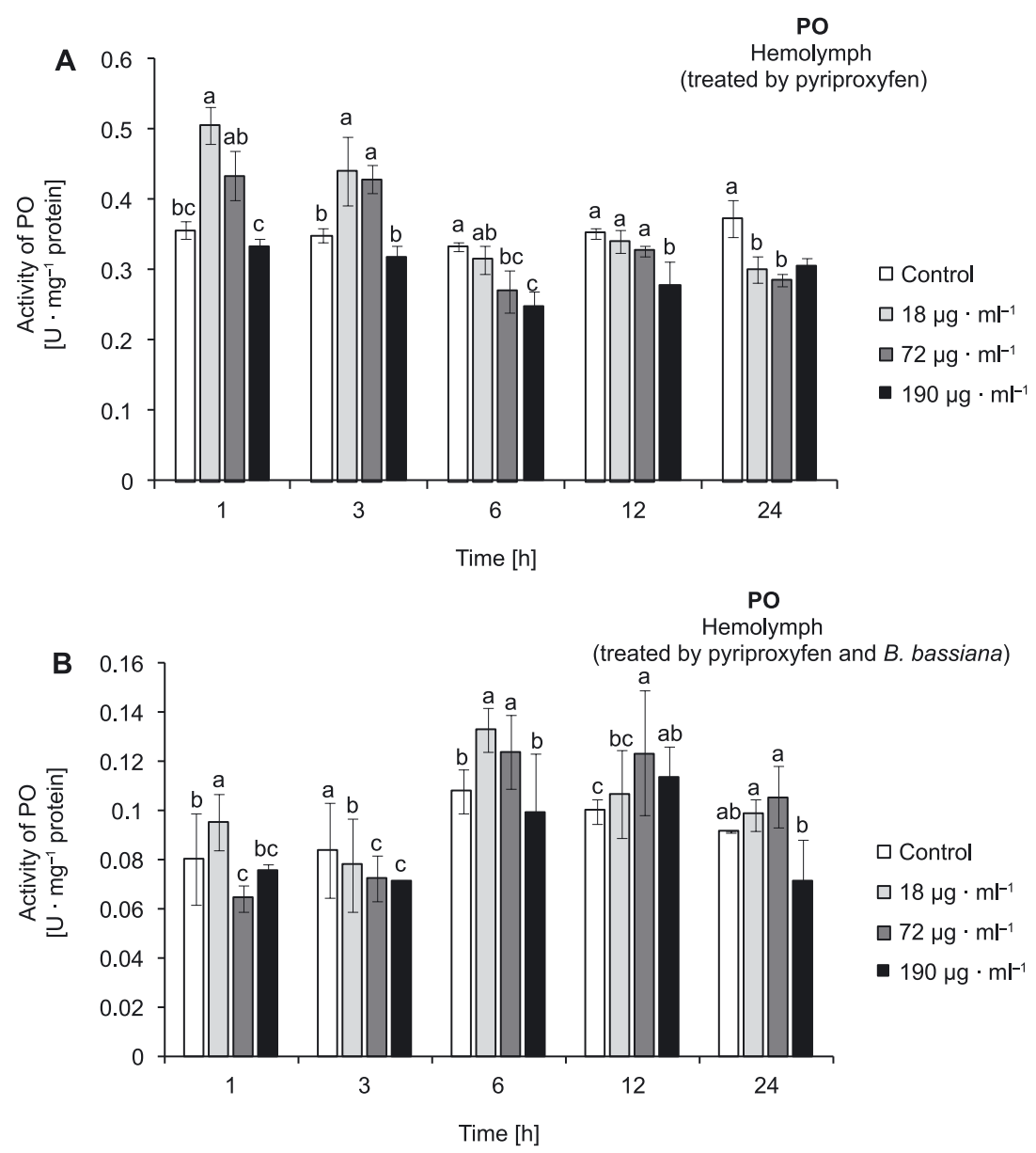

Fig. 5. Effects of pyriproxyfen (A) and pyriproxyfen + Beauveria bassiana (B) on activity of phenoloxidase (PO) in Chilo suppressalis. Statistical differences have been shown by various letters in each time intervals $(p \leq 0.05$, Tukey test) 
cal processes of $C$. suppressalis even after the increased activities of general esterases as the major hydrolysing enzymes of $\mathrm{JH}$ and its analogues (Mirhaghparast et al. 2014). Hence, pyriproxyfen or other insect growth regulators could be considered as alternatives of organophosphorous insecticides against $C$. suppressalis. Pyriproxyfen or other insect growth regulators show that selective activity on pests do not cause environmental pollution and have the lowest effects on biocontrol agents.

\section{References}

Azambuja P., Garcia E.S., Ratcliffe N.A. 1991. Aspects of classification of hemiptera hemocytes from six triatomine species. Memórias do Instituto Oswaldo Cruz 86 (1): 1-10.

Delkash-Roudsari S., Zibaee A., Bigham Z. 2015. Purification and characterization of a phenoloxidase in the hemocytes of Ephestia kuehniella Zeller (Lepidoptera: Pyralidae): effects of insect growth regulators and endogenous inhibitors. Journal of Enzyme Inhibition and Medicinal Chemistry: 1-6. DOI: 103109/14756366.2014.954107

Franssens V., Smagghe G., Simonet G., Claeys I., Breugelmans B., DeLoof A., Vanden Broeck J. 2006. 20-Hydroxyecdysone and juvenile hormone regulate the laminarin-induced nodulation reaction in larvae of the fleshfly, Neobellieria bullata. Developmental and Comparative Immunology 30 (9): 735-740.

Gelbič I., Strbáčkova J., Berger J. 2005. Influence of metyrapone on the morphology of hemocytes of the Egyptian cotton leafworm, Spodoptera littoralis (Boisd). Zoological Studies 45 (3): 371-377.

George P.J.E., Ambrose D.P. 2004. Impact of insecticides on the hemogram of Rhynocoris kumarii Ambrose and Livingstone (Hem., Reduviidae). Journal of Applied Entomology 128 (9-10): 600-604.

Goodman W.G., Granger N.A. 2005. The juvenile hormones. p. 320-408. In: "Comprehensive Molecular Insect Science" (L.I. Gilbert, ed.). Elsevier, San Diego, USA, 2568 pp.

Ishaaya I., Horowitz A.R. 1992. Novel phenoxy hormone analog (pyriproxyfen) suppresses embryogenesis and adult emergence of sweet potato whitefly. Journal of Economic Entomology 85 (6): 2113-2117.

James R.R., Xu J. 2012. Mechanisms by which pesticides affect insect immunity. Journal of Inverterbrate Pathology 109 (2): 175-182.

Kim Y., Jung S., Madanagopal N. 2008. Antagonistic effect of juvenile hormone on hemocyte-spreading behavior of Spodoptera exigua in response to an insect cytokine and its putative membrane action. Journal of Insect Physiology 54 (6): 909-915.

Lowry O.H., Rosebrough N.J., Farr A.L., Randall R.J. 1951. Protein measurement with the Folin phenol reagent. The Journal of Biological Chemistry 193: 265-275.
Mirhaghparast S.K., Zibaee A. 2013. Effects of hexaflumuron and pyriproxyfen on the purified phenoloxidase of Chilo suppressalis Walker (Lepidoptera: Crambidae). Archives of Phytopathology and Plant Protection 46 (15): 1775-1784.

Mirhaghparast S.K., Zibaee A., Hoda H. 2014. Effects of Pyriproxyfen on Intermediary Metabolism of Rice Striped Stem Borer, Chilo suppressalis Walker (Lepidoptera: Crambidae). Proceedings of the National Academy of Sciences, India Section B: Biological Sciences. 29 October 2014, Springer India.

Nijhout H.F. 1994. Insect Hormones. Princeton, University Press, Princeton, NJ, USA, 280 pp.

Rahimi V., Zibaee A., Mojahed S., Maddahi K. 2013. Effects of pyriproxyfen and hexaflumuron on cellular immunity of Ephestia kuehniella Zeller (Lepidoptera: Pyralidae). Romanian Journal of Biology-Zoology 58 (2): 151-162.

Rantala M.J., Vainikka A., Kortet R. 2003. The role of juvenile hormone in immune function and pheromone pro-duction trade-offs: a test of the immunocompetence handi-cap principle. Proceedings of the Royal Society B: Biological Sciences 270 (1530): 2257-2261.

Ramzi S., Zibaee A. 2014. Biochemical properties of different entomopathogenic fungi and their virulence against Chilo suppressalis (Lepidoptera: Crambidae) larvae. Biocontrol Science and Technology 24 (5): 597-610.

Wilson K., Thomas M.B., Blanford S., Doggett M., Simpson S.J., Moore S.L. 2002. Coping with crowds: density-dependent disease resistance in desert locusts. Proceedings of the National Academy of Sciences 99 (8): 5471-5475.

Zibaee A., Bandani A.R. 2010. Effects of Artemisia annua L. (Asteracea) on the digestive enzymatic profiles and the cellular immune reactions of the Sunn pest, Eurygaster integriceps (Heteroptera: Scutellaridae), against Beauveria bassiana. Bulletin of Entomological Research 100 (02): 185-196.

Zibaee A., Bandani A.R., Malagoli D. 2012. Methoxyfenozide and pyriproxifen alter the cellular immune reactions of Eurygaster integriceps Puton (Hemiptera: Scutelleridae) against Beauveria bassiana. Pesticide Biochemistry and Physiology 102 (1): 30-37.

Zibaee A., Malagoli D. 2014. Immune response of Chilo suppressalis Walker (Lepidoptera: Crambidae) larvae to different entomopathogenic fungi. Bulletin of Entomological Research 104 (02): 155-163.

Zibaee A., Bandani A.R., Talaei-Hassanlouei R., Malagoli D. 2009. Temperature and $\mathrm{Ca}^{2+}$ ion as modulators in cellular immunity of the Sunn pest Eurygaster integriceps Puton (Heteroptera: Scutelleridae). Entomological Research 39 (6): 364-371.

Zibaee A., Sendi J., Alinia F., Ghadamyari M., Etebari K. 2009. Diazinon resistance in different selected strains of Chilo suppressalis Walker (Lepidoptera: Pyralidae), rice striped stem borer, in the north of Iran. Journal of Economical Entomology 102 (3): 1189-1196. 